\title{
Bleomycin-induced pulmonary fibrosis in fibrinogen-null mice
}

\author{
Noboru Hattori, ${ }^{1}$ Jay L. Degen, ${ }^{2}$ Thomas H. Sisson, ${ }^{1}$ Hong Liu, ${ }^{2}$ Bethany B. Moore, ${ }^{1}$ \\ Raj G. Pandrangi, ${ }^{1}$ Richard H. Simon, ${ }^{1}$ and Angela F. Drew ${ }^{2}$ \\ ${ }^{1}$ Division of Pulmonary and Critical Care Medicine, Department of Internal Medicine, \\ University of Michigan School of Medicine, Ann Arbor, Michigan, USA \\ ${ }^{2}$ Division of Developmental Biology, Children's Hospital Research Foundation, Cincinnati, Ohio, USA
}

Address correspondence to: Richard H. Simon, 6301 Medical Sciences Research Building-3, Box 0642,

University of Michigan Health Sciences Center, Ann Arbor, Michigan 48109-0642, USA.

Phone: (734) 764-4554; Fax: (734) 764-4556; E-mail: richsimo@umich.edu.

Received for publication June 7, 2000, and accepted in revised form October 26, 2000.

Mice deleted for the plasminogen activator inhibitor-1 (PAI-1) gene are relatively protected from developing pulmonary fibrosis induced by bleomycin. We hypothesized that PAI-1 deficiency reduces fibrosis by promoting plasminogen activation and accelerating the clearance of fibrin matrices that accumulate within the damaged lung. In support of this hypothesis, we found that the lungs of $\mathrm{PAI}_{-11^{-/}}$mice accumulated less fibrin after injury than wild-type mice, due in part to enhanced fibrinolytic activity. To further substantiate the importance of fibrin removal as the mechanism by which PAI-1 deficiency limited bleomycin-induced fibrosis, bleomycin was administered to mice deficient in the gene for the $\mathrm{A} \alpha$-chain of fibrinogen $(f i b)$. Contrary to our expectation, $f i b^{-/}$mice developed pulmonary fibrosis to a degree similar to $f i b^{+/-}$littermate controls, which have a plasma fibrinogen level that is $70 \%$ of that of wild-type mice. Although elimination of fibrin from the lung was not in itself protective, the beneficial effect of PAI-1 deficiency was still associated with proteolytic activity of the plasminogen activation system. In particular, inhibition of plasmin activation and/or activity by tranexamic acid reversed both the accelerated fibrin clearance and the protective effect of PAI-1 deficiency. We conclude that protection from fibrosis by PAI-1 deficiency is dependent upon increased proteolytic activity of the plasminogen activation system; however, complete removal of fibrin is not sufficient to protect the lung.

J. Clin. Invest. 106:1341-1350 (2000).

\section{Introduction}

Extravasation of plasma through damaged alveolar walls is a common occurrence during inflammatory lung diseases (1). Tissue factor that is present within the extravascular compartment triggers the coagulation cascade, leading to fibrin deposition (2). This fibrin matrix then serves as a scaffold onto which fibroblasts migrate and produce interstitial collagens. Although this fibrotic process is beneficial in circumstances such as the repair of cutaneous wounds, collagenous obliteration of airspaces renders the damaged areas of lung permanently nonfunctional. Therefore, successful repair of the injured lung requires timely removal of the fibrin-based matrix. This scenario is also presumed to be important in fibrotic processes that occur in other organs after tissue injury.

Recent experimental work using the bleomycin model of lung injury has supported the importance of the plasminogen activation system in modulating the development of pulmonary fibrosis. When bleomycin is administered to experimental animals, a multifocal inflammatory response occurs that progresses to fibrosis (3). This experimental model has served well for studying generic processes that occur when inflamed tissues are unable to effectively repair themselves and are replaced by collagenous scars. Using the bleomycin model, we have previously found that transgenic mice with a targeted deletion of their plasminogen activator inhibitor-1 (PAI-1) gene (PAI-1-/- mice) develop less pulmonary fibrosis than do wild-type mice (4). Furthermore, intratracheal instillation of an adenoviral vector containing a urokinase-type plasminogen activator (uPA) gene reduces the amount of collagen that accumulates after bleomycin exposure in mice (5).

A leading hypothesis for the protective role of the plasminogen activation system in pulmonary fibrosis has been that it is due to the plasmin-mediated clearance of fibrin. However, results of recent studies have suggested that other activities of the plasminogen activation system could theoretically influence lung injury and repair (reviewed in refs. 6-9). For example, plasmin may contribute significantly to the proteolytic activation of growth factors, cytokines, and matrix metalloproteinases, the degradation of matrix glycoproteins, and the clearance of necrotic tissue. In addition, specific components of the plasminogen activation system may have significant biological roles aside from control of plasmin-mediated proteolysis. In this regard, the 
urokinase receptor may influence cell adhesion, cell migration, and the initiation of intracellular signaling events. Finally, plasminogen might also influence the repair process by serving as the precursor of angiostatin (10). Determining whether the critical function of the plasminogen activation system in pulmonary fibrosis is plasmin-mediated fibrin clearance or another of its many functions will assist in developing therapeutic interventions. In this study, we evaluate whether the protection caused by PAI- 1 deficiency works through a plasmin-dependent proteolytic mechanism. We also evaluate the role of fibrin by determining whether its presence is required for pulmonary fibrosis to occur.

\section{Methods}

Materials. Chloramine-T, Ehrlich's solution, collagenase, DNase, Evans blue dye, formamide, heparin, human plasmin, and tranexamic acid were purchased from Sigma Chemical Co. (St. Louis, Missouri, USA). Human fibrinogen was obtained from CalbiochemNovabiochem (La Jolla, California, USA). Human Gluplasminogen and human thrombin were purchased from American Diagnostica Inc. (Greenwich, Connecticut, USA). Texas red-conjugated BSA was obtained from Molecular Probes Inc. (Eugene, Oregon, USA). Goat anti-mouse fibrinogen antibody was purchased from Nordic Immunological Laboratories BV (Tilburg, The Netherlands). The VECTASTAIN Elite $\mathrm{ABC}$ kit was obtained from Vector Laboratories Inc. (Burlingame, California, USA).

Animals. PAI-1 $1^{-/-}$mice (11) were originally provided by P. Carmeliet (University of Leuven, Belgium). These were subsequently backcrossed to $\mathrm{C} 57 \mathrm{BL} / 6$ mice for eight generations. Wild-type C57BL/6 mice $\left(P A I-1^{+/+}\right)$ were purchased from Charles River Laboratories Inc. (Wilmington, Massachusetts, USA). A $\alpha$-chain fibrinogen-deficient $\left(\mathrm{fib}^{-/-}\right)$mice (12) and fibrinogen-heterozygotic $\left(\mathrm{fib}^{+/-}\right)$mice were produced by mating $\mathrm{C} 57 \mathrm{BL} / 6 \mathrm{fib}^{-/-}$males and $\mathrm{fib}^{+/-}$females that were inbred for six generations.

Bleomycin exposure. For each experiment, age- and weight-matched groups of mice were used. Mice were anesthetized with either intraperitoneal pentobarbital or inhalation of $2 \%$ isoflurane, and the trachea was exposed by a cervical incision. Bleomycin (Nippon Kayaku Co., Tokyo, Japan) was dissolved in PBS and then instilled intratracheally using a 27-gauge needle. The dose for $P A I-1^{-/-}$and $P A I-1^{+/+}$mice (average weight $20 \pm 2 \mathrm{~g}$ ) was $2.5 \mathrm{U} / \mathrm{kg}$. A higher dose of bleomycin $(5 \mathrm{U} / \mathrm{kg})$ was given in experiments using $\mathrm{fib}^{-/}$and $\mathrm{fib}^{+/-}$mice (average weight $27 \pm 4 \mathrm{~g}$ ) after preliminary experiments using $2.5 \mathrm{U} / \mathrm{kg}$ failed to produce consistent pulmonary fibrosis in the $\mathrm{fib}^{+/-}$mice.

Hydroxyproline assay. Hydroxyproline content in whole mouse lungs was used to quantify lung collagen content and was measured colorimetrically by a method described previously, with modifications (13). At the time of sacrifice, both lungs were removed and the extrapulmonary airways and blood vessels were excised and discarded. The lung parenchyma was homogenized in $1.0 \mathrm{ml}$ of PBS, after which $1.0 \mathrm{ml}$ of $12 \mathrm{~N} \mathrm{HCl}$ was added, and the samples were hydrolyzed at $110^{\circ} \mathrm{C}$ for 24 hours. Five microliters of each sample was mixed with 5 $\mu \mathrm{l}$ of citrate-acetate buffer (5\% citric acid, $1.2 \%$ glacial acetic acid, $7.25 \%$ sodium acetate, and $3.4 \%$ sodium hydroxide). One hundred microliters of chloramine-T solution (1.4\% chloramine-T, $10 \% N$-propanol, and $80 \%$ citrate-acetate buffer) was added, and the mixture was incubated for 20 minutes at room temperature. Ehrlich's solution was added and the samples were incubated at $65^{\circ} \mathrm{C}$ for 18 minutes. Absorbance was measured at $550 \mathrm{~nm}$. Standard curves were generated for each experiment using reagent hydroxyproline as a standard. Results were expressed as micrograms of hydroxyproline contained in total lung tissue.

Inflammatory leukocytes in bronchoalveolar lavage fluid and collagenase digests of lung tissue. Using a modification of a previously described method (14), mice were sacrificed with a lethal dose of pentobarbital, the trachea was cannulated with an 18-gauge needle, and the lungs were lavaged twice with PBS. The lavage fluid was pooled and centrifuged, and the cell pellet was resuspended in erythrocyte-lysing buffer $\left(0.829 \% \mathrm{NH}_{4} \mathrm{Cl}\right.$, $0.1 \% \mathrm{KHCO}_{3}$, and $0.0372 \% \mathrm{Na}_{2}$ EDTA, $\left.\mathrm{pH} 7.4\right)$. The lavage cells were then washed with HBSS and resuspended in RPMI 1640. Immediately after lavage, the lung vascular bed was perfused with PBS, and the lungs were excised, minced, and digested enzymatically with digestion solution (RPMI 1640, $1 \mathrm{mg} / \mathrm{ml}$ collagenase, and $30 \mathrm{U} / \mathrm{ml}$ DNase) at $37^{\circ} \mathrm{C}$ for 30 minutes. The suspension was dispersed by repeated aspiration through a 10 -ml syringe, and erythrocytes were lysed by suspending in erythrocyte-lysis buffer. The cells were then washed twice with HBSS, resuspended in RPMI 1640, and filtered through a $100-\mu \mathrm{m}$ nylon mesh. Lung leukocytes were then purified by spinning through a $20 \%$ discontinuous Percoll gradient. Aliquots of cells from bronchoalveolar lavage fluid (BALF) and lung tissue digests were stained with trypan blue, and the cells were counted using a hemocytometer. Cells were spun onto glass slides using a cytocentrifuge (Shandon Inc., Pittsburgh, Pennsylvania, USA) and were stained with Diff-Quick (Fisher Scientific International, Pittsburgh, Pennsylvania, USA). Three hundred cells were examined on each slide and the percent of each leukocyte type was recorded.

Measurement of fibrinolysis within lungs. Fibrinolysis in murine lungs was measured by a method described previously, with modifications (15). Mice were sacrificed and $1 \mathrm{ml}$ of DMEM containing fibrinogen $(1.5 \mathrm{mg} / \mathrm{ml})$, fluorescein-labeled fibrinogen $(0.1 \mathrm{mg} / \mathrm{ml})$, plasminogen $(60 \mu \mathrm{g} / \mathrm{ml})$, thrombin $(0.2 \mathrm{U} / \mathrm{ml})$, and Texas red-conjugated BSA $(0.25 \mathrm{mg} / \mathrm{ml})$ was injected intratracheally. The Texas red-conjugated BSA was included in the instilled fluid to allow for correction for any differences in dilution that may have occurred between samples during subsequent tissue processing. After the instillation, the trachea of each animal was ligated, and 
the lungs were placed into a $50-\mathrm{ml}$ tube and incubated at $37^{\circ} \mathrm{C}$ for 5 hours. The lungs were then minced, centrifuged at $10,000 \mathrm{~g}$ for 10 minutes, and the fluorescein and Texas-red levels in $20 \mu \mathrm{l}$ of supernatant were determined. To correct for differences in dilution, the level of fluorescence from fluorescein was divided by the level of fluorescence from Texas red. During each experiment, separate aliquots of instilled solution were placed into plastic tubes. The fibrin matrices in one set of tubes were allowed to remain undisturbed ( $0 \%$ lysis control), and those in another set of tubes were lysed with excess plasmin (100\% lysis control). Both sets of tubes were centrifuged, and the ratios of fluorescein to Texas-red fluorescence in the supernatants were determined. The percent of fibrin that was degraded in the lung was calculated assuming a linear relationship between the values of the $0 \%$ and $100 \%$ lysis controls.

Measurement of fibrin content of lung tissue. Fibrin deposited in murine lungs was measured by immunoblotting to detect an $80-\mathrm{kDa} \gamma-\gamma$ chain dimer fragment that was generated by terminal digestion of fibrin with reagent plasmin (16). After intravenous injection of heparin ( $1 \mathrm{U}$ per mouse) to limit postmortem clotting, mice were sacrificed and the lung vascular bed was perfused with PBS containing $500 \mathrm{U} / \mathrm{ml}$ heparin. The lungs were excised, placed in iced buffer (0.05 M Tris, $0.15 \mathrm{M} \mathrm{NaCl}$, and $500 \mathrm{U} / \mathrm{ml}$ heparin, $\mathrm{pH}$ 7.6), and homogenized. Human plasmin prepared in the same buffer was added to a final concentration of 0.32 $\mathrm{U} / \mathrm{ml}$, and the mixture was incubated at $37^{\circ} \mathrm{C}$ for 8 hours with agitation. The plasmin-digested lungs were centrifuged and the supernatant was recovered. As a positive control, mouse plasma was obtained, clotted with thrombin, and digested with human plasmin at $37^{\circ} \mathrm{C}$ for 8 hours. The plasmin-digested clot was centrifuged and the supernatant was collected. Equal volumes of lung supernatants were boiled under reducing conditions in SDS sample buffer and then separated by electrophoresis on $10 \%$ SDS-polyacrylamide gels. Proteins were electroblotted onto a nylon membrane, and the membranes were reacted with a goat anti-mouse fibrinogen antibody. The transfer membranes were then stained using the VECTASTAIN Elite ABC kit following the manufacturer's suggested protocol.

Measurement of pulmonary vascular permeability. Using a modification of a previously described method (17), Evans blue dye prepared in PBS was injected into the tail veins of mice at $20 \mathrm{mg} / \mathrm{kg}$ body weight. Mice were sacrificed after 1 hour, and serum samples were prepared from blood aspirated by cardiac puncture. After the lung vascular bed was perfused with PBS, the lungs were excised, placed in $2 \mathrm{ml}$ of formamide, and homogenized. The homogenized lungs were incubated at $60^{\circ} \mathrm{C}$ for 16 hours, followed by centrifugation and collection of the supernatant. The absorbance of the supernatants and of serum samples diluted 50 times was measured on a spectrophotometer at $620 \mathrm{~nm}$. A permeability index was calculated as the ratio of the absorbance of the lung supernatant to that of the serum.
Tranexamic acid treatment. To block the lysine binding sites on plasminogen and plasmin, tranexamic acid was administered by continuous subcutaneous infusion via osmotic pumps (model 2002; ALZA Corp., Palo Alto, California, USA) implanted under the dorsal skin of mice. The pumps were loaded to deliver $1.8 \mathrm{mg}$ of subcutaneous tranexamic acid per day. In addition, tranexamic acid was added to the drinking water at $20 \mathrm{mg} / \mathrm{ml}$ to supplement the parenteral dose (18).

Histology. Lungs were inflation-fixed with $10 \%$ neutralbuffered formalin, and the trachea was ligated. Heart and lungs were removed and fixed, en bloc, in paraffin. Four-micron sections were prepared from the blocks and stained with hematoxylin and eosin or Gomori's trichrome to detect collagen.

Immunohistochemistry. Sections were stained immunohistochemically for fibrin(ogen) with polyclonal rabbit anti-mouse fibrinogen antibodies (19), and for fibronectin with rabbit anti-human fibronectin (DAKO Corp., Carpinteria, California, USA). Bound antibody was detected using biotinylated goat anti-rabbit immunoglobulin and the VECTASTAIN Elite ABC kit (both from Vector Laboratories Inc.) using diaminobenzidine as a peroxidase substrate (Sigma Chemical Co.). Sections were counterstained with hematoxylin.

Statistics. Results are expressed as mean \pm SEM. Differences between treatment groups were analyzed using ANOVA with Fisher's PLSD test for pairwise comparisons (StatView; Abacus Concepts Inc., Berkeley, California, USA). A $P$ value of less than 0.05 was considered statistically significant.

\section{Results}

Kinetics of collagen accumulation in bleomycin-injured lungs. Eitzman and colleagues have reported that $\mathrm{PAI}-1^{-/-}$mice developed less pulmonary fibrosis than did $P A I-1^{+/+}$animals after bleomycin administration (4). To determine the kinetics of collagen accumulation, $2.5 \mathrm{U} / \mathrm{kg}$ body weight of bleomycin was instilled intratracheally into $P A I-1^{-/-}$and $P A I-1^{+/+}$mice. By 14 days after bleomycin treatment, the lung hydroxyproline content of $P A I-1^{+/+}$ mice increased $(P<0.006)$ above that of control animals receiving $\mathrm{PBS}$ alone, and continued to increase throughout the duration of the experiment (Figure 1a). The hydroxyproline content of lungs from $\mathrm{PAI}-1^{-/-}$mice receiving bleomycin increased more slowly, becoming significantly elevated above control levels on day 21 $(P<0.01)$, when it reached a plateau. At all measured time points after bleomycin delivery, the lung hydroxyproline content of $P A I-1^{-/-}$mice remained below that of $P A I-1^{+/+}$mice $(P<0.04)$. Lung hydroxyproline content of mice 28 days after receiving PBS was used as the control condition in these experiments, because we have previously found that the lung hydroxyproline content after administration of PBS is not different from that of mice receiving no manipulations (data not shown).

Survival of mice after bleomycin treatment. Twenty PAI$1^{-/-}$mice and twenty PAI-1 $1^{+/+}$mice were given $2.5 \mathrm{U} / \mathrm{kg}$ per body weight bleomycin intratracheally. After 28 

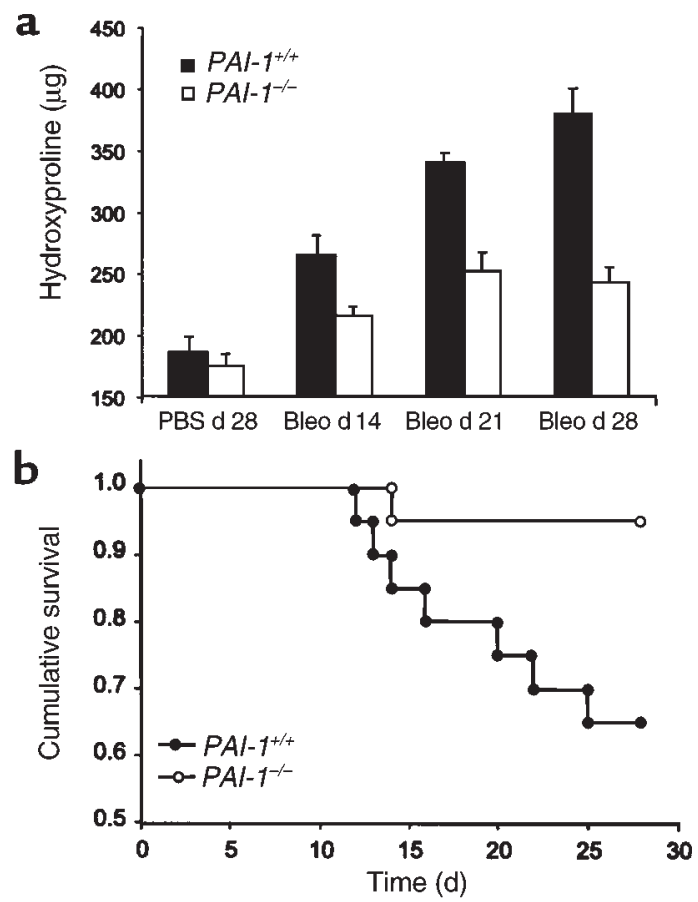

Figure 1

Effect of bleomycin on lung hydroxyproline content and survival in PAl-1-/- and PAl-1+/+ mice. Bleomycin (2.5 U/kg) or PBS was instilled intratracheally into $P A I^{-1^{-/-}}$and $P A I^{-1^{+/+}}$mice. (a) On days 14,21 , and 28 after administration, lung hydroxyproline was measured. Data are expressed as mean \pm SEM; $n=9-11$ mice per group. (b) KaplanMeier survival curve for bleomycin-exposed $\mathrm{PAl}-1^{-{ }^{-}}$and $\mathrm{PAl}-1^{+/+}$mice ( $n=20$ per group). Bleo, bleomycin.

days, when the experiment was terminated, one of the PAI-1 $1^{-/-}$mice and seven of the PAI-1 $1^{+/+}$mice had died (Figure 1b). The Mantel-Cox log rank test showed that the survival rate of the $P A I-1^{-/-}$mice was significantly better than that of the $P A I-1^{+/+}$mice $(P<0.02)$.

Lung inflammation after bleomycin injury. It is possible that the reduced collagen content of bleomycin-injured lungs in PAI-1//- mice was due to an effect of PAI-1 deficiency on the development of the inflammatory process. To evaluate this possibility, the inflammatory cell contents of BALF and collagenase-digested lung tissue were analyzed. Seven days after bleomycin delivery, when the inflammatory response is known to be maximal (20), the total number of leukocytes in the BALF and collagenase digests of lung tissue had increased equally in PAI-1-/- and $\mathrm{PAI-1^{+/+ }}$ mice (Table 1 ). The increase in percent neutrophils and decrease in percent macrophages within BALF were also similar in the two genotypes. The percent lymphocytes and eosinophils remained unchanged for both genotypes. The relative distribution of inflammatory cell types in collagenase digests of lung tissue was unchanged by bleomycin in both PAI-1-/- and $\mathrm{PAI}-1^{+/+}$animals. These quantitative assessments of leukocyte accumulation were consistent with the visual appearance of the inflammatory infiltrates seen in histological sections of lung tissue (data not shown).

Fibrin content of lungs from bleomycin-injured mice. To determine if PAI-1 deficiency decreased fibrin accumulation after lung injury, the amount of fibrin contained in lung tissue from $\mathrm{PAI}-1^{-/-}$and $\mathrm{PAI}-1^{+/+}$mice was measured 7 days and 14 days after bleomycin administration. Lung tissue was homogenized and then digested by adding excess plasmin, and the fibrin content was assessed by the intensity of the plasmindegraded $\gamma-\gamma$ dimer fragment band seen at $80 \mathrm{kDa}$ on immunoblots (21) (Figure 2a). As a positive control, fibrin formed from thrombin-treated mouse plasma was digested with plasmin and analyzed as above. The negative control sample was mouse plasma anticoagulated with ETDA. The lung digests of PBS-treated $P A I-1^{-/-}$and $P A I-1^{+/+}$mice showed no $\gamma-\gamma$ dimer fragment. On day 4 after bleomycin, the $\gamma-\gamma$ dimer fragment was easily detectable in the lungs of $P A I-1^{+/+}$ mice. The intensity of the $\gamma-\gamma$ dimer fragment band increased further, reaching a plateau on day 7 and remaining elevated on days 14 and 21 . In contrast, the intensity of the $\gamma-\gamma$ dimer fragment band from bleomycin-exposed $P A I-1^{-/-}$mice was less than that from $P A I-1^{+/+}$mice on days 4 and 7 , and had decreased further by days 14 and 21 . At the level of sensitivity used to detect the plasmin cleavage products of $\gamma-\gamma$ dimers, none were detected in lung homogenates in the absence of in vitro plasmin digestion.

Table 1

Bleomycin-induced accumulation of inflammatory cells within bronchoalveolar lavage fluid and lung tissue of PAI-1+/+ and PAI-1-/- mice

\begin{tabular}{|c|c|c|c|c|c|c|c|}
\hline & Genotype & Bleomycin & $\begin{array}{c}\text { Total cells } \\
\times 10^{5}\end{array}$ & $\begin{array}{c}\text { Mac } \\
\%\end{array}$ & $\begin{array}{c}\text { PMN } \\
\%\end{array}$ & $\begin{array}{c}\text { Lymph } \\
\%\end{array}$ & $\begin{array}{c}\text { Eos } \\
\%\end{array}$ \\
\hline BALF & $\begin{array}{l}\text { PAl-1+1++ }^{+/} \\
\text {PAl-1-1- }^{-1}\end{array}$ & $\begin{array}{l}- \\
+ \\
- \\
+\end{array}$ & $\begin{array}{l}0.29 \pm 0.03 \\
3.10 \pm 1.01 \\
0.43 \pm 0.09 \\
3.21 \pm 0.47\end{array}$ & $\begin{array}{l}97.3 \pm 0.2 \\
81.4 \pm 1.9 \\
97.2 \pm 0.2 \\
81.2 \pm 2.5\end{array}$ & $\begin{array}{r}1.1 \pm 0.4 \\
12.3 \pm 1.3 \\
1.0 \pm 0.2 \\
13.4 \pm 1.6\end{array}$ & $\begin{array}{l}1.3 \pm 0.03 \\
4.6 \pm 1.6 \\
1.9 \pm 0.51 \\
2.6 \pm 1.2\end{array}$ & $\begin{array}{l}0.2 \pm 0.1 \\
1.7 \pm 1.0 \\
0.0 \pm 0.0 \\
2.8 \pm 1.3\end{array}$ \\
\hline Lung digest & $\begin{array}{l}\text { PAl-1+/+ } \\
\text { PAl-1-1- }\end{array}$ & $\begin{array}{l}- \\
+ \\
- \\
+\end{array}$ & $\begin{array}{l}14.7 \pm 2.2 \\
27.0 \pm 1.8 \\
17.9 \pm 1.6 \\
26.8 \pm 2.0\end{array}$ & $\begin{array}{l}71.6 \pm 4.4 \\
75.1 \pm 1.7 \\
71.6 \pm 2.2 \\
75.1 \pm 1.6\end{array}$ & $\begin{array}{r}12.8 \pm 6.2 \\
9.8 \pm 1.4 \\
10.2 \pm 2.8 \\
11.6 \pm 0.2\end{array}$ & $\begin{array}{l}15.2 \pm 3.6 \\
13.9 \pm 3.8 \\
17.6 \pm 2.5 \\
12.3 \pm 1.3\end{array}$ & $\begin{array}{l}0.4 \pm 1.0 \\
1.2 \pm 1.0 \\
0.7 \pm 0.2 \\
0.9 \pm 0.04\end{array}$ \\
\hline
\end{tabular}

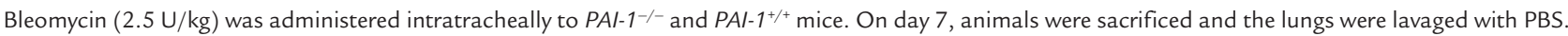
Minced lung tissue was digested with collagenase, and leukocytes were separated from residual debris by filtration and Percoll-gradient centrifugation. The leukocyte numbers and differential counts (Mac, macrophages; PMN, neutrophils; Lymph, lymphocytes; Eos, esosinophils) were determined by hemocytometer and examination of cytospin preparations, respectively. Data presented as mean $\pm \mathrm{SEM} ; n=4$. 
Lung permeability in bleomycin-exposed mice. The decreased amount of fibrin within the lungs of bleomycin-exposed PAI-1-1- mice could be due to either decreased formation of fibrin or its accelerated removal. Because fibrin is formed predominantly from the extravasation of plasma from damaged blood vessels, the effect of PAI-1 deficiency on lung vascular permeability that follows bleomycin administration was assessed. To measure permeability, mice were injected intravenously with Evans blue dye, which binds rapidly to circulating albumin. The accumulation of dye within lung tissue can be detected spectrophotometrically, thereby allowing measurement of macromolecular permeability. As shown in Figure 2b, the amount of vascular permeability 7 days after bleomycin administration was similar in $\mathrm{PAI-1^{-/- }}$ and $\mathrm{PAI-1^{+/+ }}$ mice. Of note, Figure 2 a shows that fibrin accumulation on day 7 was less in PAI-1-1/- mice than in $P A I-1^{+/+}$mice at a time when vascular permeability was similar. Thus, PAI-1 $1^{-/-}$mice appear to be able to remove lung fibrin more efficiently than $P A I-1^{+/+}$mice do. On day 14 , less dye had accumulated in the lungs of $\mathrm{PAI}-1^{-/-}$mice than in lungs of $P A I-1^{+/+}$mice. Hence, the reduced fibrin accumulation in $P A I-1^{-/-}$mice on day 14 could be due to a combination of increased fibrin clearance and/or decreased vascular permeability.

Rate of fibrin clearance from mouse lungs. To assess the capacity of the lungs to degrade fibrin within the airspaces, an in situ fibrinolytic assay was used. Plasminogen and thrombin were added to fluoresceinlabeled fibrinogen, and the mixture was immediately instilled into the lungs of mice ex vivo. After 5 hours, fibrinolysis was measured by determining the percentage of soluble fluorescent material that was soluble from lung minces. Experiments were performed on days 7 and 14 after bleomycin administration. Day-today variation in the percent fibrinolysis occurring in control mouse lungs prevented direct comparison of absolute values from experiments performed on different days. Nevertheless, on both day 7 and day 14, bleomycin-injured $P A I-1^{-/-}$mice lysed fibrin more efficiently than did $P A I-1^{+/+}$mice $(P<0.002$; Figure $2 c)$. Unexpectedly, fibrin degradation in $\mathrm{PAI}-1^{+/+}$mice was similar between those treated with PBS alone and those that were exposed to bleomycin. This is in contrast to studies of BALF where soluble fibrinolytic activity has been reported to be partially suppressed during pulmonary inflammation $(22,23)$.

Inhibition of plasmin activity with tranexamic acid. PAI-1 has multiple functions beyond inhibiting the enzymatic activity of plasminogen activators (6-9). It affects cellular adhesion and migration by interfering with the interaction between the UPA receptor and vitronectin. PAI-1 also initiates cellular uptake of the uPA receptor-uPA complex. It is possible that one or more of these other functions of PAI- 1 could be instrumental in modulating the extent of fibrosis that occurs after lung injury. To determine if PAI-1 deficiency limits collagen deposition by allowing more plasmin activation to
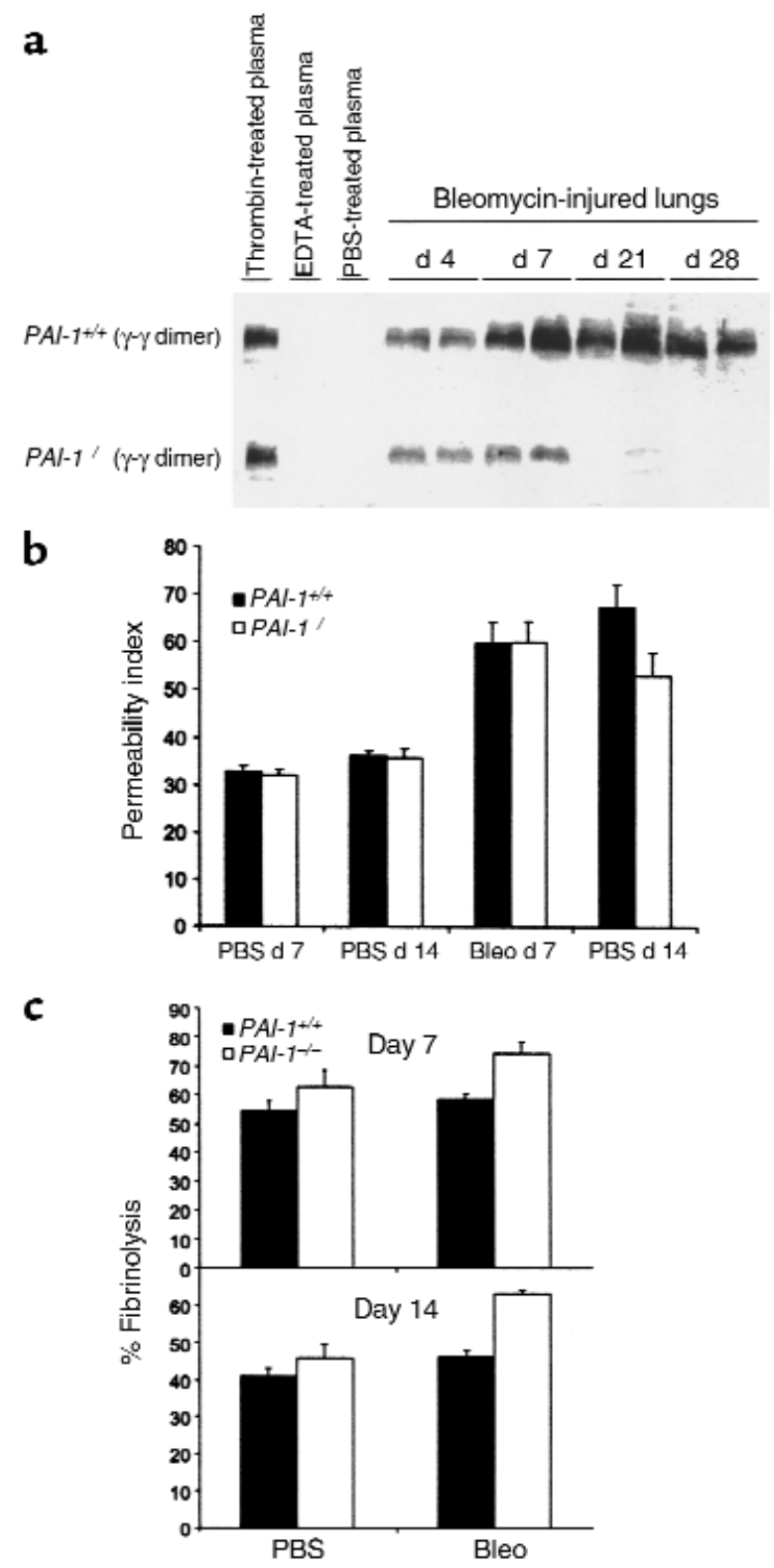

\section{Figure 2}

Dynamics of fibrin accumulation in bleomycin-exposed mice. Bleomycin $(2.5 \mathrm{U} / \mathrm{kg})$ or PBS was instilled intratracheally into PAI-1-/and $P A I^{-1+/+}$ mice. (a) To measure tissue fibrin content, lungs were harvested from heparinized mice on the days indicated and were processed as described in Methods. As positive and negative controls, fibrin samples formed within thrombin-treated plasma and anticoagulated plasma were prepared and processed in an identical manner. Samples were separated by SDS-PAGE and then immunoblotted to detect plasmin-generated, fibrin-derived $\gamma-\gamma$ dimer fragment. (b) Vascular permeability was measured 7 days and 14 days after bleomycin administration, using lung accumulation of Evans blue dye that was injected intravenously 1 hour before sacrifice. Data are expressed as mean $\pm \mathrm{SEM} ; n=7$ for bleomycin-injured mice, $n=3$ for PBS control mice. (c) The rate of fibrin degradation was measured in lungs of $\mathrm{PAl}-1^{-/-}$and $\mathrm{PAl}-1^{+/+}$mice 7 days and 14 days after intratracheal instillation of PBS or bleomycin. At the time of sacrifice, fibrin was formed within pulmonary airspaces by intratracheally instilling fluorescein-labeled fibrinogen, plasminogen, and thrombin. After 5 hours, the percent of soluble fluorescent material was measured. Data are expressed as mean $\pm \mathrm{SEM} ; n=4-7$ mice per group. 

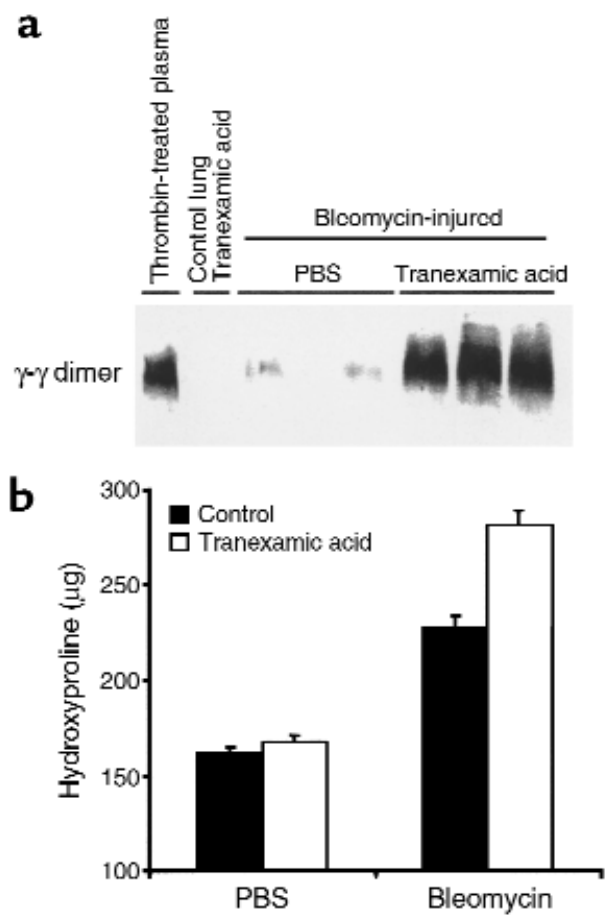

Figure 3

Effects of tranexamic acid on lungs of bleomycin-injured $P A I_{-1-1-}^{-1}$ mice. PBS or bleomycin was instilled intratracheally into PAI-1-1mice, and PBS or tranexamic acid was administered by subcutaneous osmotic pump and addition to drinking water. After 14 days, lungs were harvested and analyzed. (a) Immunoblot of fibrinderived $\gamma-\gamma$ dimer fragment in the plasmin-digested lungs of tranexamic acid-treated PAl-1-1- mice. (b) Hydroxyproline content of lung tissue. Results are expressed as mean \pm SEM; $n=5-8$ mice per bleomycin-treated group.

occur, we reversed this effect by administering tranexamic acid to $P A I-1^{-/-}$mice. Tranexamic acid inhibits plasmin proteolysis both by blocking plasmin(ogen) binding to its proteolytic substrates $(24,25)$ and by interfering with plasminogen binding to cellular receptors, where cell surface-bound uPA efficiently converts it to plasmin (26). Administration of tranexamic acid to $P A I-1^{-/-}$mice successfully reduced the plasmin activity in bleomycin-injured lungs, as indicated by an increase in lung fibrin content (Figure 3a). In the absence of bleomycin, fibrin did not accumulate within the lungs of mice receiving tranexamic acid. Importantly, this inhibition of plasmin activity by tranexamic acid caused a significant increase in lung collagen as measured by hydroxyproline content $(P<0.002$; Figure $3 \mathrm{~b})$. These results support the importance of plasmin enzymatic activity in limiting lung fibrosis after an inflammatory injury. In separate experiments discussed below, tranexamic acid was administered to bleomycin-treated animals having wild-type PAI-1 genes. No significant tranexamic acid effect was seen, indicating that normal PAI-1 expression masks any additional inhibitory effect of tranexamic acid.

Bleomycin-induced pulmonary fibrosis in mice that are genetically deficient in fibrinogen. The above results showed that PAI-1 deficiency enhances fibrinolytic activity and limits both fibrin accumulation and collagen deposition after bleomycin administration. To determine whether fibrin accumulation is a required step for bleomycin-induced fibrosis to occur, bleomycin was given to mice that were genetically deficient in fibrinogen $\left(\mathrm{fib}^{-/-}\right.$mice). These animals, having a targeted deletion within the gene coding for the $A \alpha$ chain of fibrinogen, have no circulating fibrinogen, whereas heterozygous animals $\left(\mathrm{fib}^{+/-}\right)$have a plasma fibrinogen level that is $70 \%$ that of the wild type. (Note: Fibrinogen levels of $\mathrm{fib}^{+/}$mice are reduced by less than half of wild-type levels because the synthesis of the $\mathrm{B} \beta$ chain appears to limit fibrinogen production in wild-type animals.) Contrary to our hypothesis that fibrinogen deficiency would protect from fibrosis, bleomycin caused an increase in lung hydroxyproline content in both $\mathrm{fib}^{+/-}$and $\mathrm{fib}^{-/-}$mice $(P<0.004$; Figure 4).

The similarity in lung hydroxyproline content of $\mathrm{fib}^{-/-}$ and $\mathrm{fib}^{+/-}$mice did not appear to be a consequence of selective death of mice of any one genotype after bleomycin instillation. A higher fraction of $f i b^{-/-}$mice did die in the first two days after surgery ( 17\% postsurgical mortality with either bleomycin or saline instillation) than $\mathrm{fi}^{+/-}$mice, which almost always survived the surgical trauma. Of those surviving the initial days after surgery, approximately $80 \%$ of both $f i b^{-/-}$and $f i b^{+/-}$mice survived to the day of lung collection. All mice lost $5-10 \%$ of body weight after the administration of bleomycin. Recovery of some weight occurred between 14 and 24 days, with no difference between $\mathrm{fib}^{-/-}$and $\mathrm{fib}^{+/-}$mice. PBS-treated mice in both groups also lost some weight initially after surgery, but regained weight at a faster rate than did bleomycin-treated mice $(P<0.007)$.

At 14 days and 24 days after bleomycin treatment, focal fibrotic lesions were seen in lungs of both $\mathrm{fib}^{+/-}$ and $\mathrm{fib}^{-/-}$mice (Figure 5), whereas no lesions were observed in lungs collected from saline-treated animals of either genotype. Most of the lesions con-

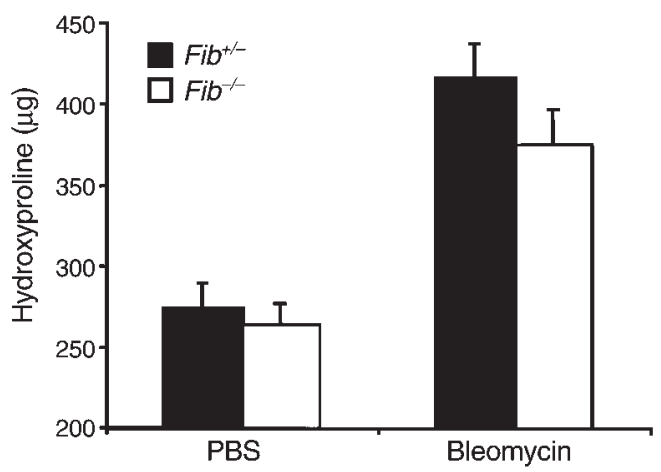

Figure 4

Effect of bleomycin $(5 \mathrm{U} / \mathrm{kg})$ on lung hydroxyproline content of $\mathrm{fib}^{-/-}$ and $\mathrm{fib}^{+/-}$mice. Bleomycin $\left(\mathrm{fib}^{+/-}, n=6\right.$; $\left.\mathrm{fib}^{-/-}, n=5\right)$ or PBS $\left(\mathrm{fib}^{+/-}\right.$, $n=6$; $\left.\mathrm{fib}^{-/-}, n=3\right)$ was instilled intratracheally into $\mathrm{fib}^{-/-}$and $\mathrm{fib}^{+/-}$ mice. Twenty-four days after administration, lung hydroxyproline was measured. Data are expressed as mean \pm SEM. 
tained some areas of fibrillar material that stained blue for collagen deposits with Gomori's trichrome stain, and were frequently associated with inflammatory cell accumulations consisting of lymphocytes and macrophages. The number of spindle-shaped cells resembling fibroblasts appeared to be increased in lesions from both genotypes. Other than the distinct absence of detectable fibrin(ogen) in lungs of $\mathrm{fib}^{-/-}$mice (Figure 5), no qualitative differences were seen in lesion composition or occurrence in either genotype. Immunohistochemical analyses for fibrin(ogen) in $\mathrm{fib}^{+/-}$mice revealed intense staining, presumably of fibrin, within lesions in which the normal alveolar architecture had been obliterated.

Immunohistology was also used to assess the distribution of fibronectin within lung tissue after bleomycin treatment. Fibronectin was chosen for study because it has cell-adhesive properties that might compensate for the absence of provisional fibrin matrices in $\mathrm{fib}^{-/-}$ mice. Furthermore, fibronectin might accumulate in $\mathrm{fib}^{-/}$animals if the absence of fibrinogen were to cause increased leaking of plasma proteins after lung injury. The intensity and pattern of fibronectin immunostaining were both indistinguishable between $\mathrm{fib}^{+/-}$and $\mathrm{fib}^{-/-}$mice (Figure 5). Fibronectin was weakly detectable within the interstitium of normal-appearing lung tissue. In contrast, prominent fibrillar staining was seen within the fibrotic lesions of $f i b^{+/-}$and $f i b^{-/-}$mice. A similar pattern was seen in wild-type and PAI$1^{-/-}$mice after bleomycin administration (data not shown).

The effect of tranexamic acid on $\mathrm{fib}^{-/-}$ mice was also studied using the same drug delivery protocol used for the PAI$1^{-/-}$mice. Contrary to what was seen in bleomycin-treated PAI-1/-/ mice, tranexamic acid in $\mathrm{fib}^{-/-}$mice caused only a minor, statistically insignificant increase in lung hydroxyproline content 14 days after bleomycin administration (Figure 6). However, tranexamic acid also failed to cause a significant increase in hydroxyproline when given to bleomycin-treated $\mathrm{ib}^{+/-}$mice. Apparently, in both $\mathrm{fib}^{-/-}$and $\mathrm{fib}^{+/-}$mice, tranexamic acid did not add substantially to the inhibitory effect that is conferred by normal PAI-1 expression.

\section{Discussion}

A relationship between the plasminogen activation system and the development of pulmonary fibrosis after lung

Figure 5 inflammation is firmly established. BALF from patients with acute respiratory distress syndrome (27, 28 ) or a variety of chronic inflammatory lung diseases (22) show suppressed fibrinolytic activity, largely due to increases in fibrinolytic inhibitors. Mice with impaired fibrinolysis caused by either overexpression of a PAI-1 transgene (4) or by targeted deletion of the plasminogen gene (29) develop increased lung fibrosis after lung inflammation. Conversely, increasing the activity of the fibrinolytic system by targeted deletion of PAI-1 genes (as investigated in this report) protects the lung from fibrosis induced by inflammation. Furthermore, increasing uPA in the lung by direct protein administration (30) or by gene transfer (5) also reduces
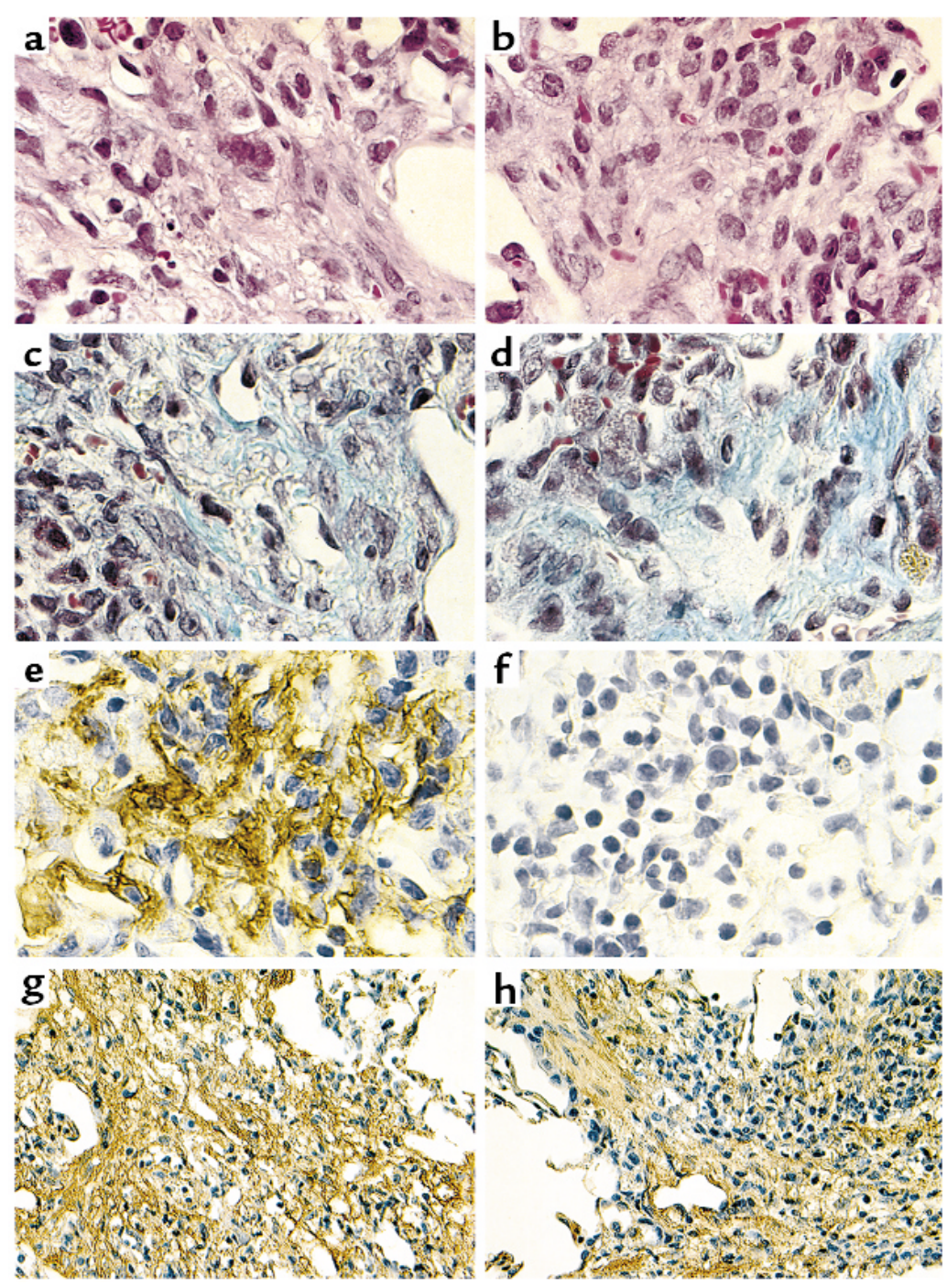

Microscopic appearance of focal fibrotic lesions in lungs of $\mathrm{fi}^{+/-}$and $\mathrm{fib}^{-/-}$mice treated with bleomycin. Fibrotic lesions with associated inflammatory infiltrates typical of those occurring in lungs of $\mathrm{fib}^{+/-}$(a) and $\mathrm{fib}^{-/-}$(b) mice 24 days after bleomycin instillation (hematoxylin and eosin staining). Gomori's trichrome stain illustrating collagen deposition within lesions of both $\mathrm{fib}^{+/-}$(c) and $\mathrm{fib}^{-/-}$(d) mice. Immunohistochemical stain showing that fibrin(ogen) is associated with lesions of $\mathrm{fib}^{+/-}$mice (e; brown reaction product) but is absent in $\mathrm{fib}^{-/-}$mice (f). Immunostaining for fibronectin shows a similar pattern in $\mathrm{fib}^{+/-}(\mathbf{g})$ and $\mathrm{fib}^{-/-}(\mathbf{h})$ mice. 


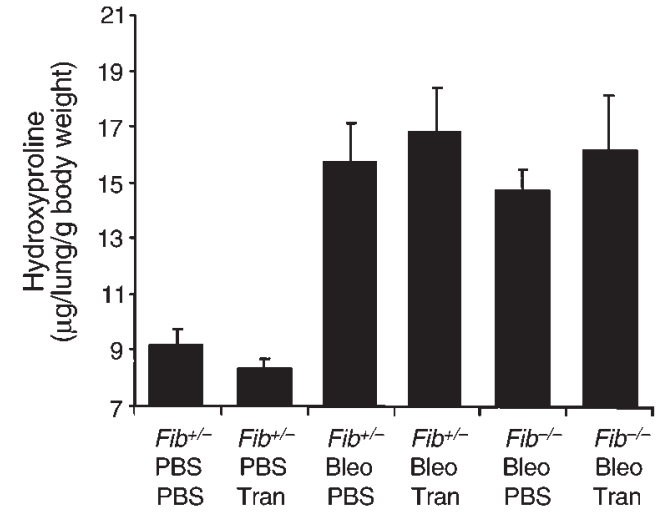

Figure 6

Effect of tranexamic acid on lung hydroxyproline content of bleomycin-treated lungs of $\mathrm{fib}^{+/-}$and $\mathrm{fib}^{-/-}$mice. PBS or bleomycin was instilled intratracheally, and PBS or tranexamic acid was administered by subcutaneous osmotic pump and addition to drinking water. After 14 days, lungs were harvested and analyzed for hydroxyproline content. Results expressed as mean \pm SEM; $n=4-7$ animals per group. Tran, tranexamic acid.

fibrosis. Importantly, these latter interventions can be delivered after the inflammatory injury has already begun, making the approach potentially useful as a treatment for pulmonary fibrotic diseases. To develop this theory, we studied the mechanisms by which PAI$1^{-/-}$mice limit the development of pulmonary fibrosis.

Our initial hypothesis was that accelerated fibrin clearance from the damaged lung would remove the provisional matrix onto which fibroblasts can migrate and form collagenous scars. In agreement with this hypothesis, fibrin was found to accumulate less in $P A I-1^{-/-}$mice after lung injury than in wild-type mice. The reduction in fibrin accumulation did not appear to be due to reduced fibrin formation because the extent of inflammatory injury and permeability leak seen 7 days after bleomycin delivery was similar in the $P A I-1^{-1-}$ mice and the wild-type mice. Instead, the reduced fibrin accumulation in $P A I-1^{-/-}$mice was probably a result of enhanced fibrinolysis, a phenomenon that was demonstrated directly by comparing the rate of degradation of fibrin matrices formed ex vivo in the lungs of $\mathrm{PAI-1^{-/- }}$ and wild-type animals. In fact, the difference that was measured in the fibrinolytic rate of lungs from $P A I-1^{-/-}$mice and wild-type mice is probably lower than the true difference in fibrinolytic capacity. The reason for the reduced difference is that the experimental matrix that was generated within the lungs was formed by instilling reagent fibrinogen, plasminogen, and thrombin. Such a matrix would minimize a major difference between wild-type and PAI-1-1- animals, namely, the presence of PAI-1 in endogenously formed matrices in wildtype mice and its absence in PAI-1-/- mice. The difference in fibrinolytic rates that we reported between the two genotypes thus depended upon the small amount of PAI-1 present in the alveolar lining fluid before the matrix-forming substrates were instilled. It is likely that the same considerations explain the similarity in fibrinolytic rates seen in wild-type mice treated with PBS or bleomycin. Forming the intra-alveolar matrices using reagent fibrinogen and thrombin would diminish the effect of increased PAI-1 production that is induced by bleomycin.

To our surprise, the experiments using $\mathrm{fib}^{-/-}$mice showed that fibrin was not required for fibrosis to occur in the injured lung. Despite the consistent presence of fibrin during inflammatory lung diseases and the ability of fibrin to serve as a provisional matrix for wound repair, fibrosis occurs in its absence. The minor difference in lung hydroxyproline content between the bleomycin-treated $\mathrm{fib}^{+/-}$and $\mathrm{fib}^{-/-}$mice was not statistically significant. In retrospect, previous observations of events occurring in nonpulmonary organs of $\mathrm{fib}^{-/}$ mice hinted at this possibility. In particular, scar tissue develops in $\mathrm{fib}^{-/-}$mice during the resolution of subcapsular hematomas that form spontaneously in the liver (12) and during the healing of full-thickness skin incisions (31). Nevertheless, the finding of pulmonary fibrosis in $\mathrm{fib}^{-/-}$mice does not exclude the possibility that excessive or prolonged fibrin deposition in the lung can contribute to fibrosis under specific conditions. For example, the increased fibrosis associated with plasminogen deficiency or inhibition of plasminogen activation could be, at least in part, driven by the cellular organization of fibrin deposits. If this theory is correct, then based on the studies presented here, genetically imposing fibrinogen deficiency in plasminogen-deficient mice would be expected to substantially reduce, but not eliminate, lung fibrosis. On the other hand, the further reduction in pulmonary fibrin that would be achieved by genetically imposing fibrinogen deficiency in PAI-1-deficient mice would probably result in little or no further reduction in fibrosis relative to mice lacking PAI- 1 alone. Furthermore, the results of this study would predict that the relative resistance of PAI-1-deficient mice to fibrosis would be maintained regardless of the presence or absence of fibrinogen. Studies are underway to directly test these working hypotheses.

Although fibrin is obviously a key physiologic target of plasmin (31), biologically significant roles of plasmin beyond fibrin clearance have been documented in specific physiological and pathological contexts. An important physiological role of plasminogen activation in hepatic repair that is independent of plasmin-mediated fibrinolysis has been recently established (9). Plasminogen-deficient mice were shown to have a marked impairment in the reparative removal of necrotic liver tissue; this impediment persisted in the absence of fibrin deposition. A role of plasmin outside fibrinolysis has also been recognized in the pathological context of kainateinduced neurodegeneration in the hippocampus (32). Detailed studies revealed that laminin is probably one critical plasmin substrate relevant to neurodegeneration. Plasmin may also limit pulmonary fibrosis by con- 
tributing to the clearance of common extracellular matrix components (reviewed in refs. 33 and 34). Alternatively, plasmin-mediated activation of protease zymogens, such as latent metalloproteinases (35), may limit pulmonary fibrosis by secondarily removing extracellular matrix proteins whose persistence would obliterate alveolar spaces. Although these proteolytic activities could theoretically cause harm by destroying normal tissue, their net effect appears beneficial during lung inflammation in $\mathrm{PAI}-1^{-/-}$mice. Additional targets of the plasminogen activation system that might be relevant to pulmonary fibrosis are latent growth factors (8). In particular, HGF is activated by plasminogen activators in vitro (36) and might promote alveolar epithelial cell proliferation and repair of damaged alveolar surfaces in vivo (37). Recent studies have shown that administration of HGF can reduce bleomycin-induced lung injury (38). Taken together, the fibrotic tendency may be modified by plasminogen activators/plasmin through combinatorial cleavage of many distinct substrates, including fibrin and nonfibrin substrates.

The beneficial effect of PAI-1 deficiency on pulmonary fibrosis could also be due to nonproteolytic features of the plasminogen activator/plasmin system. In vitro studies have shown that the binding of PAI-1 to the extracellular matrix protein vitronectin reduces the affinity of uPA receptor for vitronectin $(39,40)$. When uPA is bound to its cell-surface receptor, interaction with PAI- 1 increases the removal of $\mathrm{uPA}$ receptor complexes from the cell surface (41). Among other activities, the uPA receptor is involved in initiating intracellular signaling (42). The net in vivo effects of these complex PAI-1 interactions are difficult to predict given their potential roles in adhesion, migration, and intracellular signaling involving leukocytes, fibroblasts, epithelial cells, and endothelial cells. However, even if these nonproteolytic features are operative in limiting pulmonary fibrosis, certain observations still point strongly to a proteolytic role for the plasminogen activator/plasmin system. In particular, tranexamic acid was found to reverse the beneficial effects of PAI-1 deficiency. Tranexamic acid inhibits plasmin-mediated proteolysis by interfering with the binding of plasmin to its target substrates (24), or by preventing plasminogen binding to cellular surfaces where it can be activated efficiently by receptor-bound uPA (43). Although tranexamic acid promoted fibrosis in animals deficient in PAI-1, similar effects were not seen in animals with a normal PAI-1 genotype. Apparently, the regulatory effects of PAI-1 on plasminogen activation were not influenced significantly by the addition of tranexamic acid.

Modulation of fibrotic processes by the plasminogen activator/plasmin system is not unique to the lung. Decreased plasminogen activation was found to result in increased amounts of extracellular matrix within immune-damaged renal glomeruli (44) and synovial tissue (45), whereas increased plasminogen activation reduced the development of adhesions within an inflamed pleural space (46) and reduced capsular fibro- sis of the optic lens after complicated cataract surgery (47). From these data, a pattern emerges in which impaired plasminogen activation in multiple organ systems is generally associated with increased fibrosis, whereas interventions that enhance plasminogen activator activity are associated with less fibrosis. However, there are situations in which a different relationship is seen. For example, after myocardial infarction, uPA-deficient mice were found to accumulate less collagen in the necrotic areas (48). Notably, this decreased deposition of extracellular matrix is associated with a severe impediment in the clearance of necrotic cardiomyocytes and diminished fibroblast infiltration. The seemingly contradictory profibrotic aspect of plasminogen activation in some tissues (e.g., ischemic heart tissue) and the antifibrotic potential of increased plasminogen activator activity in other tissues (e.g., bleomycin-challenged lung tissue) might be reconciled by distinct biological features associated with different sites and types of injury. Specific features that might determine the local pro- or antifibrotic consequences of defects in plasminogen activation include (1) the presence (e.g., ischemic tissue) or absence (e.g., skin incision) of dense fields of necrotic cells; (2) the composition of the extracellular matrix; and (3) the availability and type of other proteolytic systems for clearing matrix components and cellular debris.

In conclusion, our results show that PAI-1 deficiency reduces the extent of pulmonary fibrosis that follows inflammatory lung injury. The improvement tracks closely with the proteolytic activities of the plasminogen activator/plasmin system. Despite our supposition that accelerated removal of fibrin was responsible for limiting fibrosis, fibrin is not required for fibrosis to occur. Degradation of additional substrates or activation of other antifibrotic pathways may be involved in the beneficial effect of augmenting the plasminogen activation system.

Note added in proof. Subsequent to the submission of this article, a short communication was published reporting the development of bleomycin-induced fibrosis in mice with a targeted deletion of the fibrinogen $\gamma$-chain (49).

\section{Acknowledgments}

This work was supported by NIH grants HL-46487 (R.H. Simon), HL-63194 (J.L. Degen), and HL-07749 (T.H. Sisson); and by a grant from the American Heart Association, Ohio Valley Affiliate (A.F. Drew).

\footnotetext{
1. Fein, A., et al. 1979. The value of edema fluid protein measurement in patients with pulmonary edema. Am. J. Med. 67:32-38.

2. Idell, S., et al. 1987. Bronchoalveolar lavage procoagulant activity in bleomycin-induced lung injury in marmosets. Characterization and relationship to fibrin deposition and fibrosis. Am. Rev. Respir. Dis. 136:124-133.

3. Adamson, I.Y., and Bowden, D.H. 1974. The pathogenesis of bleomycininduced pulmonary fibrosis in mice. Am. J. Pathol. 77:185-197.

4. Eitzman, D.T., et al. 1996. Bleomycin-induced pulmonary fibrosis in transgenic mice that either lack or overexpress the murine plasminogen activator inhibitor-1 gene. J. Clin. Invest. 97:232-237.

5. Sisson, T.H., Hattori, N., Xu, Y., and Simon, R.H. 1999. Treatment of bleomycin-induced pulmonary fibrosis by transfer of urokinase-type plasminogen activator genes. Hum. Gene Ther. 10:2315-2323.

6. Chapman, H.A. 1997. Plasminogen activators, integrins, and the coordi-
} 
nated regulation of cell adhesion and migration. Curr. Opin. Cell Biol. 9:714-724.

7. Carmeliet, P., and Collen, D. 1998. Development and disease in proteinase-deficient mice: role of the plasminogen, matrix metalloproteinase and coagulation system. Thromb. Res. 91:255-285.

8. Rifkin, D.B., Mazzieri, R., Munger, J.S., Noguera, I., and Sung, J. 1999. Proteolytic control of growth factor availability. APMIS. 107:80-85.

9. Bezerra, J.A., et al. 1999. Plasminogen deficiency leads to impaired remodeling after a toxic injury to the liver. Proc. Natl. Acad. Sci. USA. 96:15143-15148

10. O'Reilly, M.S., et al. 1994. Angiostatin: a novel angiogenesis inhibitor that mediates the suppression of metastases by a Lewis lung carcinoma. Cell. 79:315-328.

11. Carmeliet, P., et al. 1993. Plasminogen activator inhibitor-1 gene-deficient mice. I. Generation by homologous recombination and characterization. J. Clin. Invest. 92:2746-2755.

12. Suh, T.T., et al. 1995. Resolution of spontaneous bleeding events but failure of pregnancy in fibrinogen-deficient mice. Genes Dev. 9:2020-2033.

13. Woessner, J.F. 1961. The determination of hydroxyproline in tissue and protein samples containing small proportions of this amino acid. Arch. Biochem. Biophys. 93:440-447.

14. Curtis, J.L., et al. 1994. Experimental murine pulmonary cryptococcosis. Differences in pulmonary inflammation and lymphocyte recruitment induced by two encapsulated strains of Cryptococcus neoformans. Lab. Invest. 71:113-126.

15. Hattori, N., Sisson, T.H., Xu, Y., and Simon, R.H. 1999. Upregulation of fibrinolysis by adenovirus-mediated transfer of urokinase-type plasminogen activator genes to lung cells in vitro and in vivo. Hum. Gene Ther. 10:215-222.

16. Lawson, C.A., et al. 1997. Monocytes and tissue factor promote thrombosis in a murine model of oxygen deprivation. J. Clin. Invest. 99:1729-1738.

17. Green, T.P., Johnson, D.E., Marchessault, R.P., and Gatto, C.W. 1988. Transvascular flux and tissue accrual of Evans blue: effects of endotoxin and histamine. J. Lab. Clin. Med. 111:173-183.

18. Bastaki, M., et al. 1997. Basic fibroblast growth factor-induced angiogenic phenotype in mouse endothelium. A study of aortic and microvascular endothelial cell lines. Arterioscler. Thromb. Vasc. Biol. 17:454-464.

19. Drew, A.F., et al. 1998. Ligneous conjunctivitis in plasminogen-deficient mice. Blood. 91:1616-1624.

20. Janick-Buckner, D., Ranges, G.E., and Hacker, M.P. 1989. Alteration of bronchoalveolar lavage cell populations following bleomycin treatment in mice. Toxicol. Appl. Pharmacol. 100:465-473.

21. Olman, M.A., et al. 1996. Polymerization of fibrinogen in murine bleomycin-induced lung injury. Am. J. Physiol. 271:L519-L526.

22. Chapman, H.A., Allen, C.L., and Stone, O.L. 1986. Abnormalities in pathways of alveolar fibrin turnover among patients with interstitial lung disease. Am. Rev. Respir. Dis. 133:437-443.

23. Idell, S., James, K.K., Gillies, C., and Fair, D.S. 1989. Abnormalities of pathways of fibrin turnover in lung lavage of rats with oleic acid and bleomycin-induced lung injury support alveolar fibrin deposition. Am.J. Pathol. 137:387-389.

24. Hoylaerts, M., Lijnen, H.R., and Collen, D. 1981. Studies on the mechanism of the antifibrinolytic action of tranexamic acid. Biochim. Biophys. Acta. 673:75-85.

25. Moser, T.L., Enghild, J.J., Pizzo, S.V., and Stack, M.S. 1993. The extracellular matrix proteins laminin and fibronectin contain binding domains for human plasminogen and tissue plasminogen activator. J. Biol. Chem. 268:18917-18923

26. Plow, E.F., Herren, T., Redlitz, A., Miles, L.A., and Hoover-Plow, J.L. 1995. The cell biology of the plasminogen system. FASEB J. 9:939-945.

27. Idell, S., et al. 1989. Local abnormalities in coagulation and fibrinolytic pathways predispose to alveolar fibrin deposition in the adult respiratory distress syndrome. J. Clin. Invest. 84:695-705.
28. Bertozzi, P., et al. 1990. Depressed bronchoalveolar urokinase activity in patients with adult respiratory distress syndrome. N. Engl. J. Med. 322:890-897.

29. Swaisgood, C.M., French, E.L., Noga, C., Simon, R.H., and Ploplis, V.A. 2000. The development of bleomycin-induced pulmonary fibrosis in mice deficient for components of the fibrinolytic system. Am. J. Pathol. 157:177-187.

30. Hart, D.A., Whidden, P., Green, F., Henkin, J., and Woods, D.E. 1994. Partial reversal of established bleomycin-induced pulmonary fibrosis by rhurokinase in a rat model. Clin. Invest. Med. 17:69-76.

31. Bugge, T.H., et al. 1996. Loss of fibrinogen rescues mice from the pleiotropic effects of plasminogen deficiency. Cell. 87:709-719.

32. Tsirka, S.E., Bugge, T.H., Degen, J.L., and Strickland, S. 1997. Neuronal death in the central nervous system demonstrates a non-fibrin substrate for plasmin. Proc. Natl. Acad. Sci. USA. 94:9779-9781.

33. Saksela, O., and Rifkin, D.B. 1988. Cell-associated plasminogen activation: regulation and physiological functions. Annu. Rev. Cell Biol. 4:93-126.

34. Vassalli, J.D., Sappino, A.P., and Belin, D. 1991. The plasminogen activator/plasmin system. J. Clin. Invest. 88:1067-1072.

35. Carmeliet, P., et al. 1997. Urokinase-generated plasmin activates matrix metalloproteinases during aneurysm formation. Nat. Genet. 17:439-444.

36. Naldini, L., et al. 1992. Extracellular proteolytic cleavage by urokinase is required for activation of hepatocyte growth factor/scatter factor. $E M B O$ J. 11:4825-4833.

37. Panos, R.J., Patel, R., and Bak, P.M. 1996. Intratracheal administration of hepatocyte growth factor/scatter factor stimulates rat alveolar type II cell proliferation in vivo. Am. J. Respir. Cell Mol. Biol. 15:574-581.

38. Yaekashiwa, M., et al. 1997. Simultaneous or delayed administration of hepatocyte growth factor equally represses the fibrotic changes in murine lung injury induced by bleomycin. A morphologic study. Am. J. Respir. Crit. Care Med. 156:1937-1944.

39. Wei, Y., et al. 1994. Identification of the urokinase receptor as an adhesion receptor for vitronectin. J. Biol. Chem. 269:32380-32388.

40. Deng, G., Curriden, S.A., Wang, S., Rosenberg, S., and Loskutoff, D.J. 1996. Is plasminogen activator inhibitor- 1 the molecular switch that governs urokinase receptor-mediated cell adhesion and release? J. Cell Biol. 134:1563-1571.

41. Cubellis, M.V., Wun, T.C., and Blasi, F. 1990. Receptor-mediated internalization and degradation of urokinase is caused by its specific inhibitor PAI-1. EMBO J. 9:1079-1085.

42. Resnati, M., et al. 1996. Proteolytic cleavage of the urokinase receptor substitutes for the agonist-induced chemotactic effect. EMBO J. 15:1572-1582

43. Plow, E.F., Freany, D.E., Plescia, J., and Miles, L.A. 1986. The plasminogen system and cell surfaces: evidence for plasminogen and urokinase receptors on the same cell type. J. Cell Biol. 103:2411-2420.

44. Kitching, A.R., et al. 1997. Plasminogen and plasminogen activators protect against renal injury in crescentic glomerulonephritis. J. Exp. Med. 185:963-968.

45. Busso, N., et al. 1998. Exacerbation of antigen-induced arthritis in urokinase-deficient mice. J. Clin. Invest. 102:41-50.

46. Strange, C., Baumann, M.H., Sahn, S.A., and Idell, S. 1995. Effects of intrapleural heparin or urokinase on the extent of tetracycline-induced pleural disease. Am. J. Respir. Crit. Care Med. 151:508-515.

47. Heiligenhaus, A., et al. 1998. Recombinant tissue plasminogen activator in cases with fibrin formation after cataract surgery: a prospective randomised multicentre study. Br. J. Ophthalmol. 82:810-815.

48. Heymans, S., et al. 1999. Inhibition of plasminogen activators or matrix metalloproteinases prevents cardiac rupture but impairs therapeutic angiogenesis and causes cardiac failure. Nat. Med. 5:1135-1142.

49. Poplis, V.A., et al. 2000. A total fibrinogen deficiency is compatible with the development of pulmonary fibrosis in mice. Am. J. Pathol. 157:703-708 